\title{
Stress Induced Effects for Advanced Polarization Control in Silicon Photonics Components
}

\author{
D.-X. Xu, ${ }^{1}$ W. N. Ye, ${ }^{2}$ S. Janz,, ${ }^{1}$ A. Delâge, ${ }^{1}$ P. Cheben, ${ }^{1}$ B. Lamontagne, ${ }^{1}$ E. Post, ${ }^{1}$ and P. Waldron ${ }^{1}$ \\ ${ }^{1}$ Institute for Microstructural Sciences, National Research Council Canada (NRC), Ottawa, ON, Canada K1A OR6 \\ ${ }^{2}$ Department of Electronics, Carleton University, 1125 Colonel By Drive, Ottawa, ON, Canada K1S 5B6
}

Correspondence should be addressed to D.-X.Xu, danxia.xu@nrc-cnrc.gc.ca

Received 24 January 2008; Accepted 11 March 2008

Recommended by D. Lockwood

\begin{abstract}
We review the use of the oxide cladding stress-induced photoelastic effect to modify the polarization dependent properties in silicon-on-insulator (SOI) waveguide components, and highlight characteristics particular to this high index contrast (HIC) systems. The birefringence in SOI waveguides has its origin in the electromagnetic boundary conditions at the waveguide boundaries, and can be further modified by the presence of stress in the waveguiding materials. With typical stress levels in $\mathrm{SiO}_{2}$ films, which are often used as the upper cladding, the waveguide effective index can be altered anisotropically up to the order of $10^{-3}$ for ridges with heights ranging from $1 \mu \mathrm{m}$ to $5 \mu \mathrm{m}$. This effect can be used effectively to counter the waveguide geometrical birefringence, allowing the waveguide cross-section profiles to be optimized for design criteria other than null geometrical birefringence. Design strategies are developed for using stress engineering to achieve a variety of functions. Polarization insensitive arrayed waveguide gratings (AWGs), polarization insensitive ring resonators, and polarization splitters and filters are demonstrated using these design principles.
\end{abstract}

Copyright $\odot 2008$ D.-X. Xu et al. This is an open access article distributed under the Creative Commons Attribution License, which permits unrestricted use, distribution, and reproduction in any medium, provided the original work is properly cited.

\section{INTRODUCTION}

Silicon photonics is a rapidly growing research field with many important advances in recent years [1-9]. Primarily motivated by the potential of high-integration density and compatibility with mature CMOS technologies, siliconon-insulator (SOI) has been the main material platform for silicon photonic waveguide components $[1,9]$. Along with these benefits, control and utilization of polarizationdependent properties arise as a challenge, as well as open new possibilities in advanced designs and functionalities [10-15].

Polarization-dependent properties have long been an important issue in integrated optics systems. In applications such as wavelength demultiplexing and high resolution spectroscopy, the shift in channel wavelength with the polarization state of the incoming optical signal often compromises the device spectral resolution [8]. One approach to handle this issue is to produce devices with polarization insensitive performance. In some cases this approach may not be practical, and then polarization diversity may be adopted where the signal is divided into orthogonal polarization states using a polarization splitter and processed separately. In this paper, we review the characteristics of modal birefringence in SOI waveguides, with regard to the two main sources of polarization anisotropy, namely, the geometrical and the stress-induced birefringence [13, 16]. Design methodologies and experimental demonstrations for using the stress-induced birefringence to achieve polarization-insensitive arrayed waveguide gratings (AWGs) $[13,17]$, polarization insensitive ring resonators $[18,19]$, and zero-order AWG-based broadband polarization splitters [20] are presented.

\section{WAVEGUIDE BIREFRINGENCE: GEOMETRICAL EFFECTS}

In planar waveguides, the modal birefringence (the difference in the effective index for the TM and TE polarizations $\left.\Delta n_{\text {eff }}=n_{\text {eff }}^{\mathrm{TM}}-n_{\text {eff }}^{\mathrm{TE}}\right)$ results from the combination of geometrical, compositional, and stress-induced effects. In low index contrast glass waveguides, the geometrical effect is negligible and the birefringence is primarily controlled by the residual stress in the waveguide layers. A large body of research has been devoted to this subject, where the main 


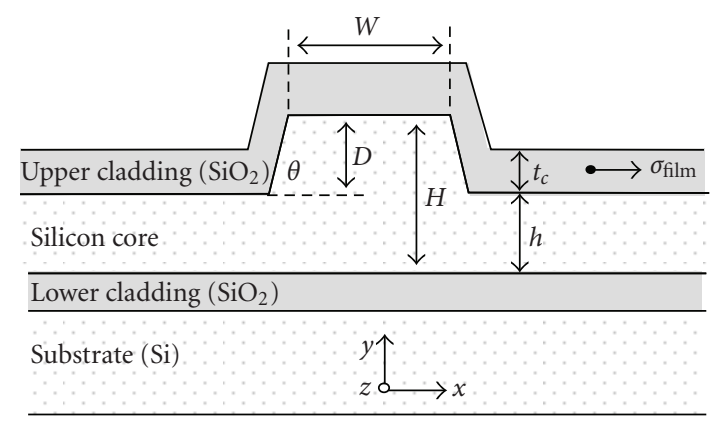

FIGURE 1: Schematic cross-section of an SOI ridge waveguide.

goal is to reduce the stress anisotropy in the waveguide core region by adjusting the thermal expansion coefficients of the cladding and core layers or creating stress relief features $[21,22]$. In high-index contrast waveguides such as SOI where the light confinement is strong, electromagnetic boundary conditions dictated by the cross-section geometry of the waveguides have the largest impact on the waveguide effective index and consequently the birefringence. These geometrical dependencies have been used to obtain single mode waveguides with large cross-sections, and to adjust the waveguide geometrical birefringence $\Delta n_{\text {geo }}$ to desired values. In an SOI ridge waveguide as illustrated in Figure 1, it is possible to minimize the birefringence by tailoring the waveguide aspect ratio, particularly for waveguides with relatively large cross-sections [10-13]. As the core size is reduced, typically to $\sim 2 \mu \mathrm{m}$ and less, it becomes increasingly difficult to attain the designed birefringence values $[13,16$, 17]. Since ridge dimensions also determine the number of waveguide modes, the minimum usable bend radius, the mode size, and the coupling between adjacent waveguides, it is often impossible to meet these different design objectives simultaneously.

As an example, Figure 2(a) (dashed lines) shows the dependence of waveguide geometrical birefringence $\Delta n_{\text {geo }}$ on the etch depth $D$ for waveguides with vertical sidewalls, ridge height $H=2.2 \mu \mathrm{m}$, and widths of 1.6 and $2.5 \mu \mathrm{m}$, respectively. Results for waveguides under stress (solid lines) will be discussed in Section 3. For a given waveguide width $W$, the polarization degeneracy $\left(\Delta n_{\text {geo }}=0\right)$ may be achieved at a specific value of etch depth $D$. At the birefringence-free point for $W=1.6 \mu \mathrm{m}$ near $D=1.3 \mu \mathrm{m}$ the change of $\Delta n_{\text {geo }}$ with the fluctuation in the etch depth is $\sim 1.5 \times 10^{-4}$ for $\Delta D= \pm 10 \mathrm{~nm}$. When $W=2.5 \mu \mathrm{m}, \Delta n_{\text {geo }}$ is less sensitive to the fluctuations in the etch depth $\left(\sim 2 \times 10^{-5}\right.$ for $\Delta D=$ $\pm 10 \mathrm{~nm}$ near $D=1.5 \mu \mathrm{m}$ ), however the birefringence-free condition cannot be achieved. To reduce the birefringence below the level required for the state-of-the-art photonic devices $\left(\Delta n_{\text {eff }}<10^{-4}\right)$ by selecting and attaining the ridge aspect ratio $W / D$, dimension control on the order of $10 \mathrm{~nm}$ is required for these waveguides $[13,16,17]$, which is very difficult in fabrication.

The effect of changing the waveguide width on the geometrical birefringence $\Delta n_{\text {geo }}$ is shown in Figure 2(b) (dashed curves), for sidewall angles of $90^{\circ}$ and $54^{\circ}$, which represent the profiles typically obtained by dry and anisotropic wet etching, respectively. Waveguide sidewall angles strongly affect the mode properties, especially as the sidewall approaches vertical. With decreasing ridge width for $\theta=90^{\circ}, \Delta n_{\text {geo }}$ shifts towards positive values initially, until the waveguide is so narrow that the modes mainly reside in the etched slab section and the birefringence changes to negative values rapidly. The birefringence of a trapezoidal ridge waveguide is less susceptible to changes in the ridge geometry (width and etch depth) as compared to a rectangular waveguide (i.e., $\theta=90^{\circ}$ ) with similar cross-section area, as can be observed from the data for a sidewall angle of $54^{\circ}$ shown in Figure 2(b). The geometrical birefringence, however, remains negative for the entire waveguide width range. For the rectangular waveguides, an optimal width can be found to satisfy the birefringence-free condition (zero birefringence at $W \sim 2 \mu \mathrm{m}$ ). Detailed discussions on the influence of geometrical parameters can be found in previous publications $[16,17]$.

Even though waveguide geometries satisfying both the single-mode and birefringence-free conditions can be found in principle, it is important to be able to implement the designs using existing fabrication technologies. Using contact-print lithography, waveguide-width variations on the order of $100 \mathrm{~nm}$ can be expected. Using state-of-theart high resolution lithography methods such as e-beam direct write or deep UV steppers, dimensional control on the order of $10 \mathrm{~nm}$ is possible but still very challenging. These techniques, however, are expensive and are not widely available. Because it is difficult to precisely control the waveguide dimensions due to limitations of the fabrication techniques, methods of controlling birefringence other than modifying waveguide geometries are desirable.

\section{PHOTOELASTIC EFFECT AND THE STRESS-INDUCED BIREFRINGENCE}

Cladding layer such as silicon dioxide is usually deposited or grown at elevated temperatures on the silicon substrate. A (compressive) stress is often found in the silicon dioxide $\left(\mathrm{SiO}_{2}\right)$ films, due to the different thermal expansion coefficients of $\mathrm{Si}\left(3.6 \times 10^{-6} \mathrm{~K}^{-1}\right)$ and oxide $\left(5.4 \times 10^{-7} \mathrm{~K}^{-1}\right)$ as well as intrinsic stress that may exist in the oxide film. When a material is subjected to a stress, the material refractive index is altered due to the photoelastic effect. The stressinduced changes in the material refractive index are given by $[13,14,23]$

$$
\begin{aligned}
& n_{x}-n_{0}=-C_{1} \sigma_{x}-C_{2}\left(\sigma_{y}+\sigma_{z}\right), \\
& n_{y}-n_{0}=-C_{1} \sigma_{y}-C_{2}\left(\sigma_{z}+\sigma_{x}\right) .
\end{aligned}
$$

Here $\sigma_{i}(i=x, y, z)$ is the stress tensor, $n_{\mathrm{i}}$ is the material refractive index in the corresponding directions, $n_{0}$ is the (isotropic) refractive index in the absence of stress, and $C_{1}$ and $C_{2}$ are the stress-optic constants. If a stress (tensor) with axial anisotropy is imposed on an originally optically isotropic material, a material birefringence is induced:

$$
n_{y}-n_{x}=\left(C_{2}-C_{1}\right)\left(\sigma_{y}-\sigma_{x}\right) .
$$




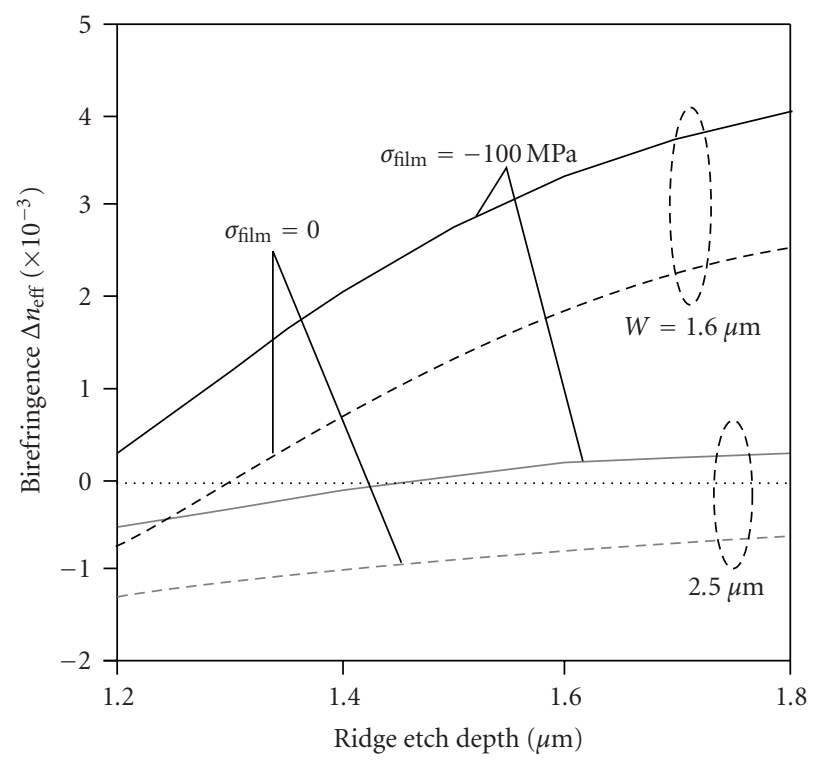

(a)

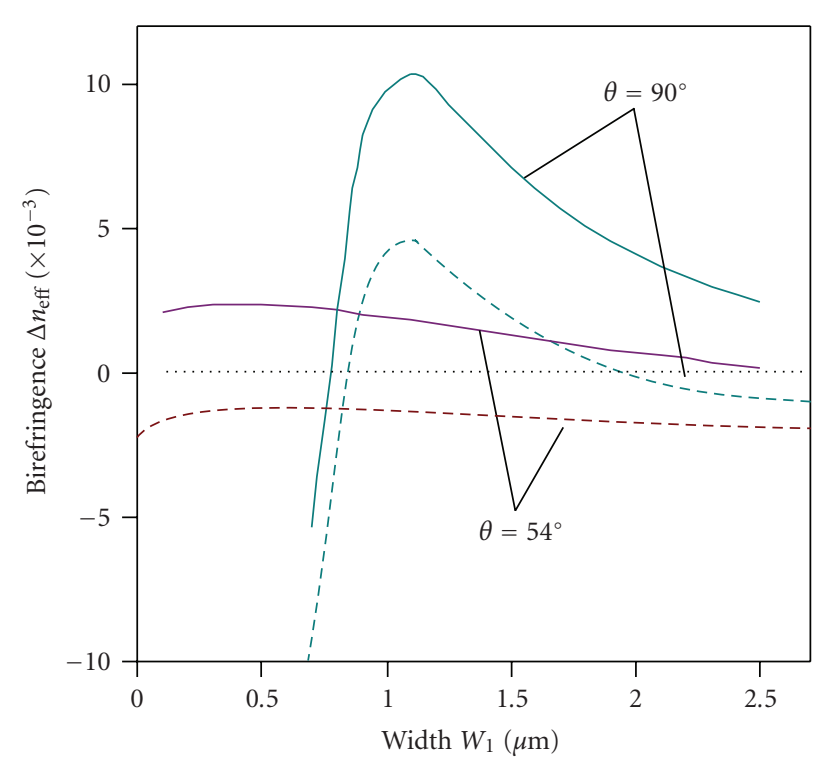

(b)

FIGURE 2: (a) Calculated modal birefringence $\Delta n_{\text {eff }}$ for waveguides with $H=2.2 \mu \mathrm{m}$. (a) $\Delta n_{\text {eff }}$ in ridge waveguides with vertical sidewalls $\left(\theta=90^{\circ}\right)$ as a function of etch depth $D$. Waveguide width $W=1.6$ and $2.5 \mu \mathrm{m}$, cladding thickness $t_{\mathrm{c}}=0.7 \mu \mathrm{m}$, and film stress $\sigma_{\text {film }}=$ $-100 \mathrm{MPa}$. (b) $\Delta n_{\text {eff }}$ in vertical $\left(\theta=90^{\circ}\right)$ and trapezoidal $\left(\theta=54^{\circ}\right)$ waveguides as a function of the ridge top width $W_{1}$, for $D=1.5 \mu \mathrm{m}$, $t_{c}=0.7 \mu \mathrm{m}$, and $\sigma_{\text {film }}=-300 \mathrm{MPa}$. The dashed curves show the geometrical birefringence $\Delta n_{\text {geo }}$ (i.e., in the absence of stress), the solid curves show $\Delta n_{\text {eff }}$ including both the geometrical and stress-induced components.

TABLE 1: Stress-optic constants of some materials.

\begin{tabular}{lccc}
\hline Material & $\lambda(\mu \mathrm{m})$ & $C_{1}\left(10^{-12} \mathrm{~Pa}^{-1}\right)$ & $C_{2}\left(10^{-12} \mathrm{~Pa}^{-1}\right)$ \\
\hline $\mathrm{Si}$ & 1.15 & -11.04 & 4.04 \\
Silica & 0.633 & 0.65 & 4.5 \\
GaAs & 1.15 & -18.39 & -10.63 \\
\hline
\end{tabular}

Stress-optic constants of silicon and glass are listed in Table 1, as well as those of GaAs for comparison.

The stress-induced material birefringence in turn causes a stress contribution $\Delta n_{\mathrm{s}}$ to the modal birefringence. The total modal birefringence can be expressed as $\Delta n_{\mathrm{eff}}=n^{\mathrm{TM}}-$ $n^{\mathrm{TE}}=\Delta n_{\text {geo }}+\Delta n_{\mathrm{s}}$. The stress distributions in an SOI ridge waveguide are shown in Figures 3(a) and 3(b), for $\mathrm{SiO}_{2}$ upper and lower claddings under a compressive stress of $\sigma_{\text {film }}=$ $-320 \mathrm{MPa}\left(\sigma_{\mathrm{film}}\right.$ is the in-plane stress component in the uniform cladding film far from the ridge), calculated using a finite-element multiphysics solver (FEMLAB). The primarily in-plane stress (Figure 3, $x$-direction) in the oxide film compresses the Si ridge and results in a compressive stress in the $x$-direction (Figure 3(a)) and a higher tensile stress in the $y$ direction (Figure 3(b)) in the silicon core. For the waveguide example shown in Figure $3, \sigma_{x} \approx-70 \mathrm{MPa}$ and $\sigma_{y} \approx$ $180 \mathrm{MPa}$ at the waveguide center. This stress anisotropy is the fundamental reason for the stress-induced birefringence. The corresponding local material birefringence $\left(n_{y}-n_{x}\right)$ is shown in Figure 3(c), with values as large as $4 \times 10^{-3}$. These modifications in the material index cause the modal index $n_{\mathrm{eff}}^{\mathrm{TM}}$ to increase and $n_{\text {eff }}^{\mathrm{TE}}$ to decrease with the oxide thickness and the stress level (Figure 4(a)). The corresponding stress-induced birefringence thus can be controlled with two parameters: the oxide thickness $t_{\mathrm{c}}$ and the film stress level $\sigma_{\text {film }}$, as shown in Figure 4(b). The stress-induced index variations resulting from commonly used oxide cladding films are of comparable magnitude to the geometrical birefringence found in typical SOI ridge waveguides. Depending on the specific value of the geometrical birefringence $\Delta n_{\text {geo }}$, the total modal birefringence may be designed to be zero or other desired values. Figure 5 shows the calculated results for maximum birefringence that can be induced by a cladding with $\sigma_{\text {film }}=-300 \mathrm{MPa}$ in waveguides with $2 \mu \mathrm{m}$ ridge height and different aspect ratios. These characteristics are the bases for stress engineering in SOI waveguides, and they can be advantageously employed in many applications.

\section{EXPERIMENTAL IMPLEMENTATIONS}

In the following, we present several examples of using stress engineering for the control of polarization-dependent properties of SOI waveguide devices. First, we present polarization insensitive AWG demultiplexers, where the birefringence in the waveguide array is eliminated by the proper selection of the oxide cladding stress and thickness $[13,17]$. Then we introduce polarization insensitive ring resonators using multimode interference (MMI) couplers to achieve polarization insensitive coupling between the bus waveguide and the ring cavity [18, 19]. Stress-induced birefringence is used to compensate the round-trip phase difference between the two polarizations. The last example is a polarization splitter in a zero-order AWG configuration, where a prism-shaped oxide patch is placed in the arrayed waveguide section to disperse the TE and TM modes into 


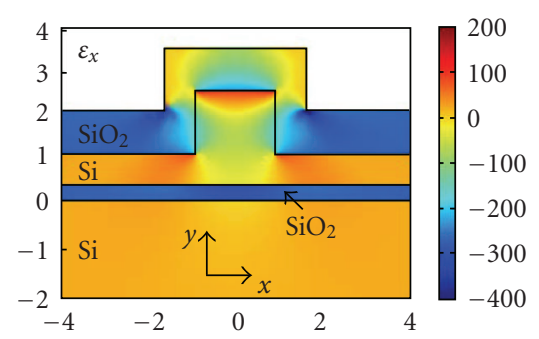

(a)

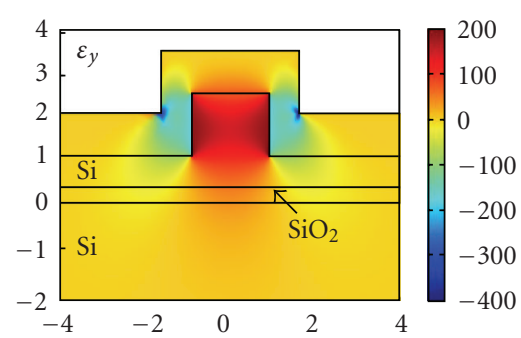

(b)

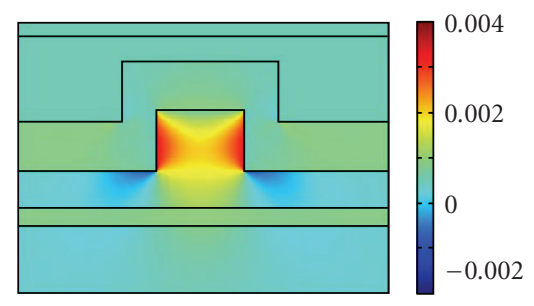

(c)

Figure 3: Stress distributions in the (a) $x$-direction and (b) $y$-direction. The ridge height is $2.2 \mu \mathrm{m}$, the width is $1.8 \mu \mathrm{m}$, and the etch depth is $1.47 \mu \mathrm{m}$. The cladding oxide is $1 \mu \mathrm{m}$ thick with a compressive stress of $\sigma_{\text {film }}=-320 \mathrm{MPa}$. (c) Stress-induced material birefringence $\left(n_{y}-n_{x}\right)$ corresponding to the stress distributions shown in Figures 3(a) and 3(b).

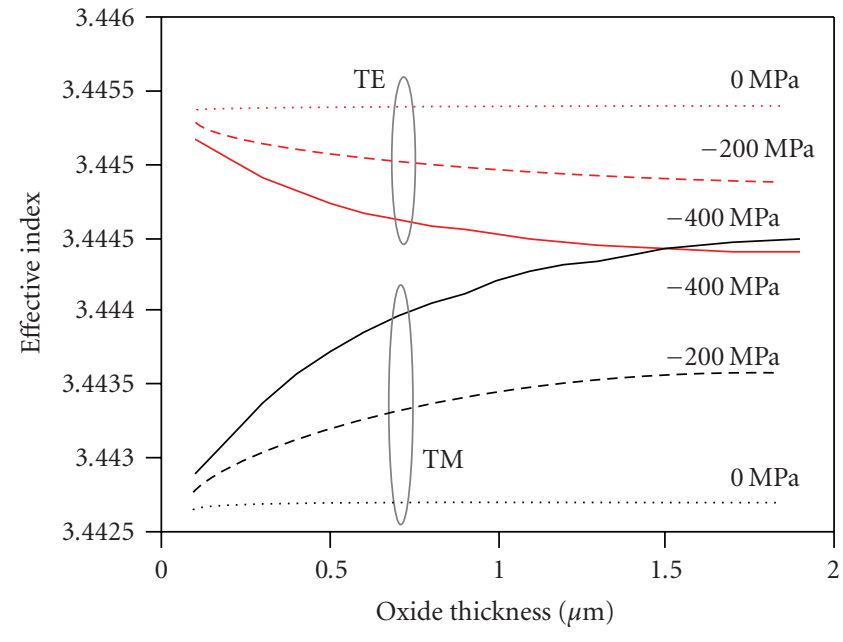

(a)

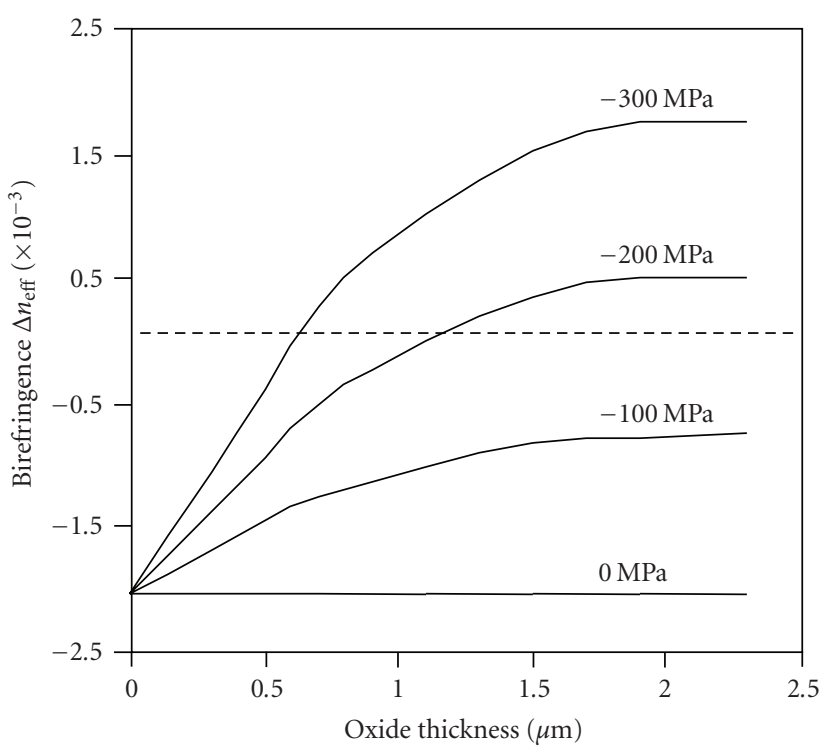

(b)

FIGURE 4: (a) Effective index as a function of the oxide upper-cladding thickness $t_{\mathrm{c}}$, for different cladding stress levels $\sigma_{\text {film }}$ of 0 , -200 , and $-400 \mathrm{MPa}$, respectively; (b) total modal birefringence $\Delta n_{\text {eff }}$ as a function of $t_{\mathrm{c}}$ and $\sigma_{\text {film }}$.

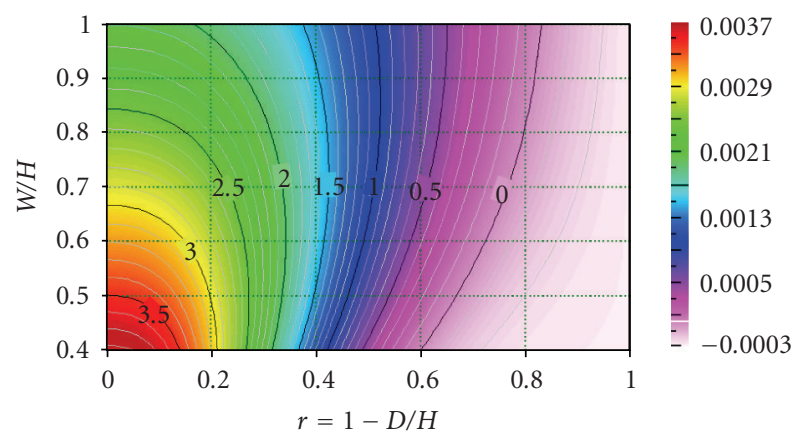

FIGURE 5: Stress-induced modal birefringence $\Delta n_{\mathrm{s}}$ for ridge waveguides with different aspect ratios, with $H=2 \mu \mathrm{m}, t_{\mathrm{c}}=2 \mu \mathrm{m}$, and $\sigma_{\text {film }}$ $=-300 \mathrm{MPa}$.

separate output waveguides. Since this AWG operates at near zero-order, broadband performance is achieved [20].

\subsection{Polarization-independent AWGs}

In AWGs, the polarization-dependent wavelength shift $\Delta \lambda=$ $\lambda_{\mathrm{TM}}-\lambda_{\mathrm{TE}}$ mainly arises from the birefringence of the arrayed waveguides and can be expressed as $\Delta \lambda \approx \lambda \Delta n_{\mathrm{eff}} / n_{\mathrm{g}}$, where $n_{\mathrm{g}}$ is the waveguide group index and $\lambda$ is the operating wavelength in vacuum. The AWGs used for this demonstration of polarization compensation using stress engineering were made on SOI substrates with a silicon thickness of $2.2 \mu \mathrm{m}$, with 9 output channels spaced at $200 \mathrm{GHz}$, and centered at $1550 \mathrm{~nm}[8,13,17]$ (see Figure 6(a)). The arrayed waveguide gratings of order 40 were formed by 100 waveguides of width $2 \mu \mathrm{m}$. Waveguides were produced using both wet and dry etching methods, yielding $\sim 54^{\circ}$ and nearly vertical sidewall angles, respectively. Waveguide birefringence simulations were performed using measured waveguide dimensions. Upper cladding oxide films of different thicknesses were deposited at $\sim 400^{\circ} \mathrm{C}$ using PECVD, with a film stress of $\sigma_{\text {film }}=-320 \mathrm{MPa}$ in the blank films, measured from wafer bowing. Figure 6(b) shows the calculated and measured dependence of $\Delta \lambda$ on the cladding thickness $t_{c}$, for both trapezoidal and rectangular waveguides. Calculated results agree well with experiments for both types of waveguides, showing that $\Delta \lambda$ can be modified over $\sim 2 \mathrm{~nm}$ wavelength range [16]. Figures $6(\mathrm{c})$ and $6(\mathrm{~d})$ show the TE and TM spectra of a wet-etched AWG before and after 


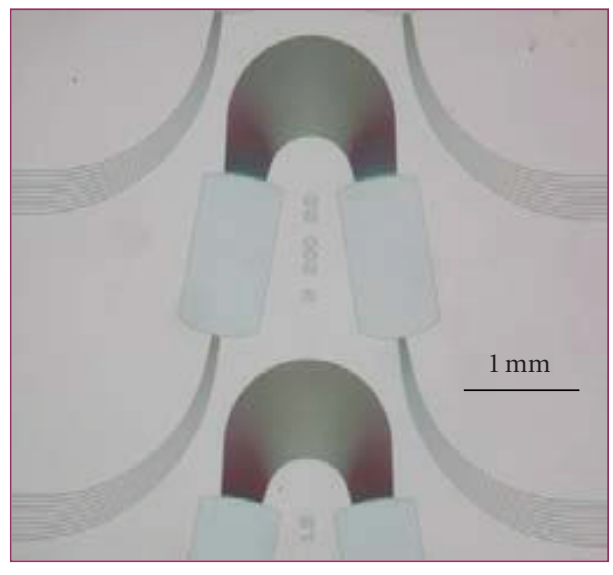

(a)

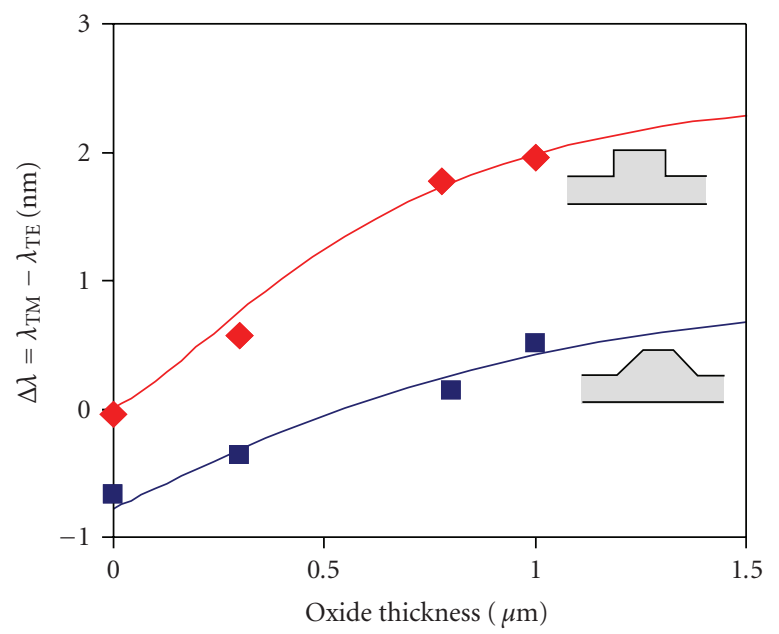

- Experiment Theory

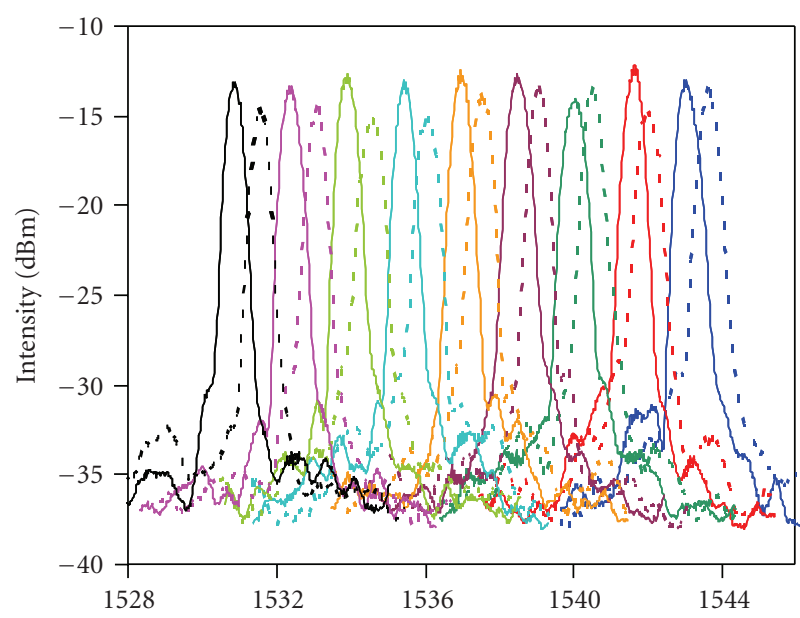

(c)

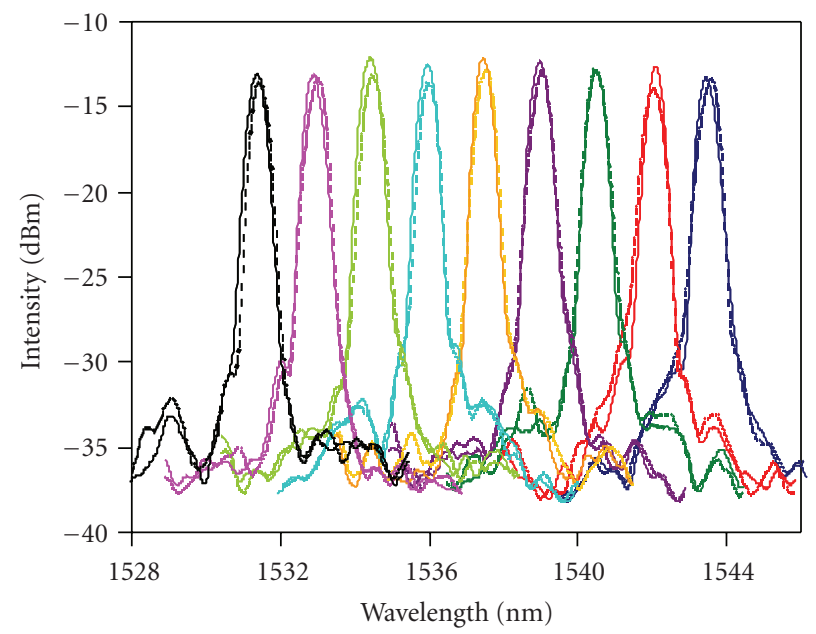

(d)

Figure 6: (a) Optical image of a fabricated AWG. (b) Polarization-dependent wavelength shift $\Delta \lambda$ as a function of the cladding thickness $t_{\mathrm{c}}$, for AWGs fabricated using wet (waveguide top width $W_{1}=1.1 \mu \mathrm{m}$ and bottom width $\left.W_{2}=3.8 \mu \mathrm{m}\right)$ and dry $\left(W_{1}=1.85 \mu \mathrm{m}\right.$ and $W_{2}=2.0 \mu \mathrm{m}$ ) etching to a depth of $D=1.47 \mu \mathrm{m}$. The cladding stress is $\sigma_{\text {film }}=-320 \mathrm{MPa}$. Measured spectra for an SOI AWG. (c) Measured spectrum for an SOI AWG without the oxide upper cladding. (d) Compensated using a $0.6 \mu \mathrm{m}$ thick $\mathrm{SiO}_{2}$ claddings with $\sigma_{\text {film }}=-320 \mathrm{MPa}$. TM (solid) and TE (dashed).

polarization compensation. The adjacent channel cross-talk is less than $-25 \mathrm{~dB}$. The noncompensated AWG device has a polarization-dependent wavelength shift of $\Delta \lambda \sim 0.6 \mathrm{~nm}$ (Figure 6(c)), arising from the geometrical birefringence of $\Delta n_{\text {geo }} \sim-1.3 \times 10^{-3}$ in the arrayed waveguide. With an oxide upper cladding film of $-320 \mathrm{MPa}$ in stress and $0.6 \mu \mathrm{m}$ in thickness, the $\Delta \lambda$ was then reduced to below $0.04 \mathrm{~nm}$ for all channels (corresponding to $\Delta n_{\text {eff }}<1 \times 10^{-4}$ ) (Figure 6(d)). The polarization dependent loss (PDL) was also found to be negligible in these devices after the compensation.

\subsection{MMI-coupled polarization-independent ring resonators}

We also applied the stress engineering technique to achieve polarization-insensitive ring resonators $[18,19]$. Ring res- onators have been used as the building blocks in applications including add-drop, switching, modulation, and sensing [24-27]. However, polarization sensitivity is often an obstacle that limits their practical use $[12,15,18]$.

The power transmission in the bus waveguide of a single coupler ring resonator (Figure 7(a)) can be obtained by a generalization of the ring resonator equations to incorporate the coupler loss $[18,28]$ :

$$
|T|^{2}=\alpha_{\mathrm{MMI}}^{2}\left[\frac{\alpha^{2}-2 \alpha t \cos \phi+t^{2}}{1-2 \alpha t \cos \phi+\alpha^{2} t^{2}}\right],
$$

where $\phi$ is the total round trip phase accumulation, and $\alpha^{2}=\alpha_{\text {MMI }}^{2} \cdot \alpha_{\text {ring }}^{2}$ is the combined power loss factor including both the ring loss and the coupler loss. The self-coupling 


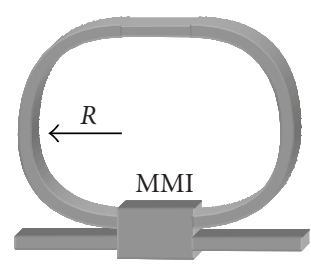

(a)

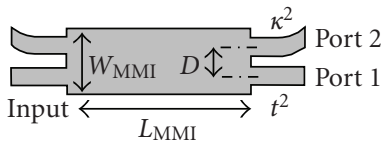

(b)
FIGURE 7: (a) A ring resonator with an MMI coupler. (b) A schematic of the MMI structure.

coefficient $t^{2}$ is the ratio of power transfer from the input to the output port 1 of the multimode interference (MMI) coupler, as indicated in Figure 7(b). The resonance extinction ratio is maximum at $\alpha^{2}=t^{2}$, which is referred to as the critical coupling condition. A series of transmission minima occur at wavelengths $\lambda_{m}$ for which the round-trip phase accumulation is integer multiple of $2 \pi$. It is apparent that a polarization dependence in the coupling coefficient $t$ and/or the loss factor $\alpha$ lead to different resonance quality factors and linewidths for TE and TM input polarizations. If the waveguides are birefringent, the round trip phase accumulation $\phi$ varies with the polarization, and the resonance wavelengths and free spectral range (FSR) are polarization sensitive. To achieve polarization independence in a ring resonator, all these factors need to be addressed.

Polarization-independent ring resonator designs using directional couplers have been investigated [12], but showed long coupler length and very stringent fabrication requirements. We proposed the use of MMI couplers to achieve polarization-independent light transfer between the resonator bus and the ring waveguides (Figures $7(\mathrm{a})$ and $7(\mathrm{~b})$ ). For simplicity, we have chosen the commonly used $2 \times 2$ restricted interference coupler with a 50:50 splitting ratio between the two output ports [29]. The coupler width is $W_{\text {MMI }}=3 D$, where $D$ is the center-to-center separation between the access waveguides (Figure $7(\mathrm{~b})$ ). The resonators are designed with the following ring and access waveguide dimensions: ridge height $H=1.5 \mu \mathrm{m}$, width $W=1.5 \mu \mathrm{m}$ and $1 \mu \mathrm{m}$, and etch depth $H-h=0.9 \mu \mathrm{m}$. A separation of $D=$ $2.5 \mu \mathrm{m}$ between the access waveguides is used in the MMI design, based on the considerations of a compact footprint and ease of fabrication, yielding an MMI width of $W_{\mathrm{MMI}}$ $=7.5 \mu \mathrm{m}$. The quality factor of a resonator is determined by the coupler splitting ratio and the loss in the resonator cavity. MMI designs with variable splitting ratios have been proposed and demonstrated by other groups and may be used for high-quality factor resonators [30, 31].

The optical field distribution in the $2 \times 2$ MMI coupler is shown in Figure 8(a), while Figure 8(b) shows the MMI coupler transfer characteristics in terms of the normalized power coupled to the fundamental mode in ports 1 and 2, respectively, as a function of the coupler length. For each polarization, a splitting ratio close to 50:50 is achieved over the range of coupler length $L_{\mathrm{MMI}}$ from 82 to $86 \mu \mathrm{m}$, with a coupler imbalance of $\leq 0.05 \mathrm{~dB}$. The combined power in the two outputs is the total transmitted power $\alpha_{\mathrm{MMI}}^{2}$, which indicates the loss through the coupler (with $\alpha_{\text {MMI }}^{2}$ $=1$ being lossless). The optimum coupling length differs by $\sim 2.5 \mu \mathrm{m}$ (or $\sim 3 \%$ ) between the two polarizations. As a first approximation, a coupler length of $L_{\mathrm{MMI}}=84 \mu \mathrm{m}$ at the crossing point is used in all our resonator designs, where a similar excess loss of $\sim-0.2 \mathrm{~dB}$ is found for both polarizations. This choice is based on the assumption that losses from all other mechanisms are similar for TE and TM, which is not necessarily the case. In addition to the coupler excess loss, there are several other mechanisms contributing to the overall resonator loss. For example, the waveguide propagation loss due to scattering depends on the optical mode overlap with the roughness at the waveguide boundaries and is generally polarization dependent. This loss is proportional to the propagation distance, and therefore to the cavity length of a ring resonator. Another source is the radiation loss in waveguide bends, as well as possible losses at discontinuities such as the junctions between the straight and curved waveguides. These factors need to be considered in the device design, and detailed discussions can be found in [19].

Since the stress in SOI waveguides caused by the cladding layer can be used effectively to control the birefringence over a wide range, it can be applied to eliminate the polarization dependence in the ring phase accumulation $\phi$. The phase change in the MMI section is $2 \pi n_{\mathrm{eff}}^{\mathrm{MMI}} L^{\mathrm{MMI}} / \lambda$, plus a constant correction factor which is a multiple integer of $\pi / 2$ [32]. Since the stress-induced birefringence in the ring waveguide $\Delta n_{\mathrm{s}}^{\text {ring }}$ is much larger than that in the wider MMI section [16] (i.e., $\Delta n_{\mathrm{s}}^{\mathrm{MMI}} \ll \Delta n_{\mathrm{s}}^{\text {ring }}$ ), the difference in the phase accumulation $\Delta \phi$ between TM and TE polarizations can be expressed as [18]:

$$
\Delta \phi=\frac{2 \pi}{\lambda}\left[\Delta n_{\text {geo }}^{\mathrm{MMI}} L_{\mathrm{MMI}}+\Delta n_{\mathrm{geo}}^{\text {ring }} L_{1}+\Delta n_{\mathrm{s}}^{\text {ring }} L_{1}\right]
$$

Here $L_{1}$ is the total length of the narrower ring waveguide sections, $L_{1}=L-L_{\mathrm{MMI}}=2 \pi R+L_{\mathrm{MMI}}$. Figure 9 shows the ring resonator phase difference $\Delta \phi=\phi^{\mathrm{TM}}-\phi^{\mathrm{TE}}$ as a function of the oxide cladding thickness for two cladding stress levels. Obviously, various combinations of cladding stress and thickness can be found to adjust $\Delta \phi$ to zero.

We have fabricated MMI-based ring resonators in SOI wafers with $1.5 \mu \mathrm{m}$ thick silicon at the Canadian Photonics Fabrication Center (CPFC). SEM images of a fabricated device are shown in Figures 10(a) and 10(b). Standard inhouse PECVD process produces oxide films with $-250 \mathrm{MPa}$ stress. Measured transmission spectra for a MMIcoupled ring resonator with a radius of $200 \mu \mathrm{m}$ are shown in Figure 10(c) for TE and TM polarizations. Over the $4 \mathrm{~nm}$ scan range, the polarization-dependent wavelength shift is below $2 \mathrm{pm}$, which is the laser scan step used in these measurements. The measured free spectral range (FSR) is $\sim 0.46 \mathrm{~nm}$, and the quality factor is $Q \sim 15,000$. Polarization insensitive operation is achieved in these resonators, demonstrating the effectiveness of the proposed method for polarization control. 


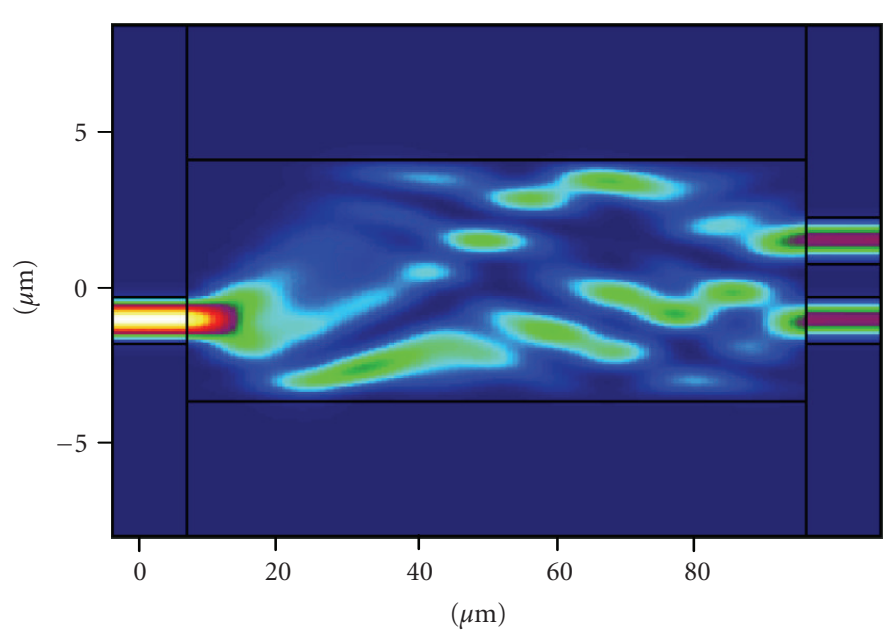

(a)

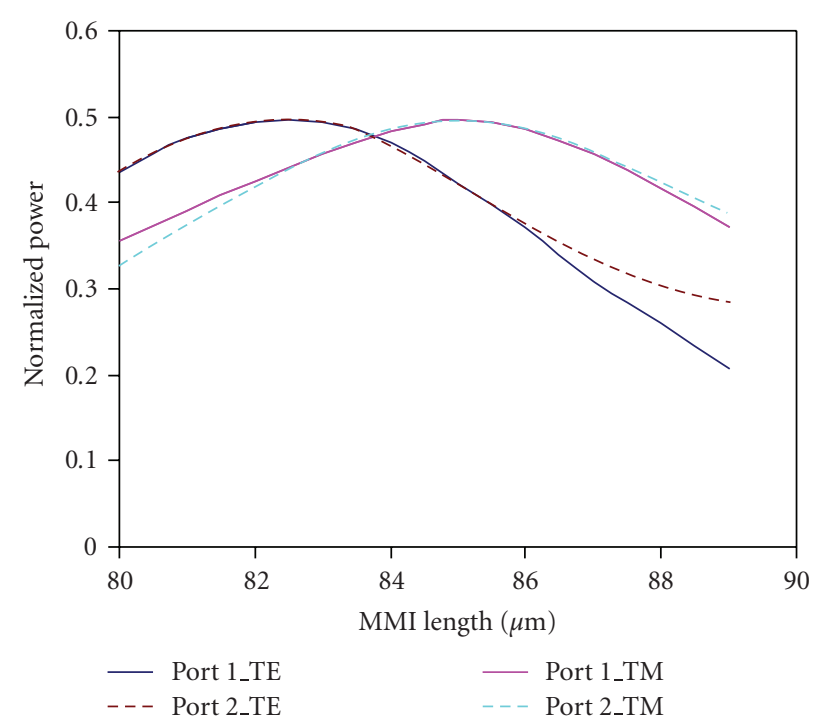

(b)

Figure 8: (a) Optical field distribution in the SOI MMI coupler of $7.5 \mu \mathrm{m} \times 84 \mu \mathrm{m}$ in dimensions, with an etch depth of $1 \mu \mathrm{m}$. (b) Corresponding normalized power in the fundamental mode in ports 1 and 2, for the TE and TM polarizations.

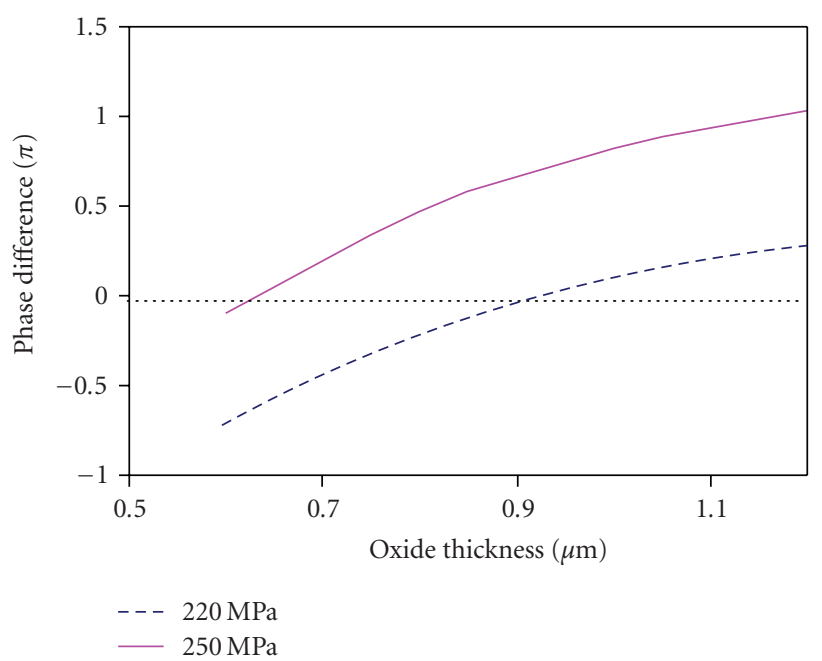

FIGURE 9: Phase difference $\Delta \phi=\phi^{\mathrm{TM}}-\phi^{\mathrm{TE}}$ as a function of the oxide cladding thickness for an MMI-coupled ring resonator with a radius $R$ of $200 \mu \mathrm{m}$ and an oxide cladding stress of -220 and $-250 \mathrm{MPa}$, respectively.

\subsection{Broadband polarization splitter in a zero-order AWG configuration}

Stress-induced effects can also be used to produce high level of birefringence in selected waveguide areas. Since the stressinduced index modifications are several orders of magnitude smaller than the core-cladding refractive index contrast in $\mathrm{SOI}$, the effect of a $\mathrm{SiO}_{2}$ cladding on mode shape is negligible. Thus there is little mode mismatch loss or polarizationdependent loss at the junctions between waveguide sections with and without the cladding. Figure 11 shows a zeroorder AWG where all of the arrayed waveguides have identical geometrical path lengths [20]. A triangular patch of oxide cladding is placed in the arrayed waveguide section with a constant length increment $\Delta L$ between the adjacent waveguides. The stress in the waveguide cladding induces a polarization-dependent phase difference in the light signal propagating in the waveguide array (see Figure 4(a)), causing a polarization-dependent tilt in the phase front of the light in the free propagation region (FPR) (see Figure 11(b)). Since all waveguides have the same physical length, the phase difference for light propagating in the adjacent waveguides of the arrayed section depends solely on the cladding stressinduced index change and the patch-length increment. Since the stress-induced index change in the TE and TM polarization modes has opposite signs for silicon waveguides, the two modes are spatially displaced in the opposite directions in the focal region relative to the free propagation region centerline, as shown in Figure 11(b). With proper placement of the two receiver waveguides along the focal plane, the two polarizations are spatially separated.

Zero-order AWG-based polarization splitters were designed and fabricated on SOI wafers, with a $2.2 \mu \mathrm{m}$ thick silicon core layer. The deposited oxide cladding film had a thickness of $1 \mu \mathrm{m}$, and the measured cladding film stress was approximately $-340 \mathrm{MPa}$. The overall device size is $\sim 12 \mathrm{~mm} \times 4 \mathrm{~mm}$. Figure 12(a) shows several fabricated AWG devices with triangular cladding regions of varied patch sizes. Based on the measured cladding film stress and thickness, the cladding patch-length increment $\Delta L$ between the adjacent waveguides was set to $16.4 \mu \mathrm{m}$ to produce the required polarization-dependent phase front tilt at the output of the waveguide array. Since stress-induced effects do not vary with wavelength appreciably, and the AWG operates close to zero order, the performance of the splitter is expected to be wavelength independent over a large application bandwidth, which was experimentally 


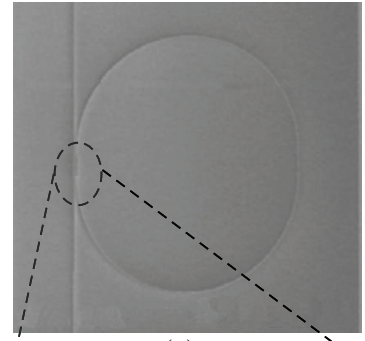

(a)

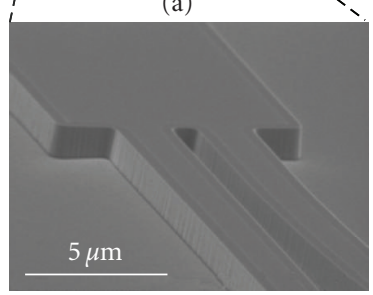

(b)

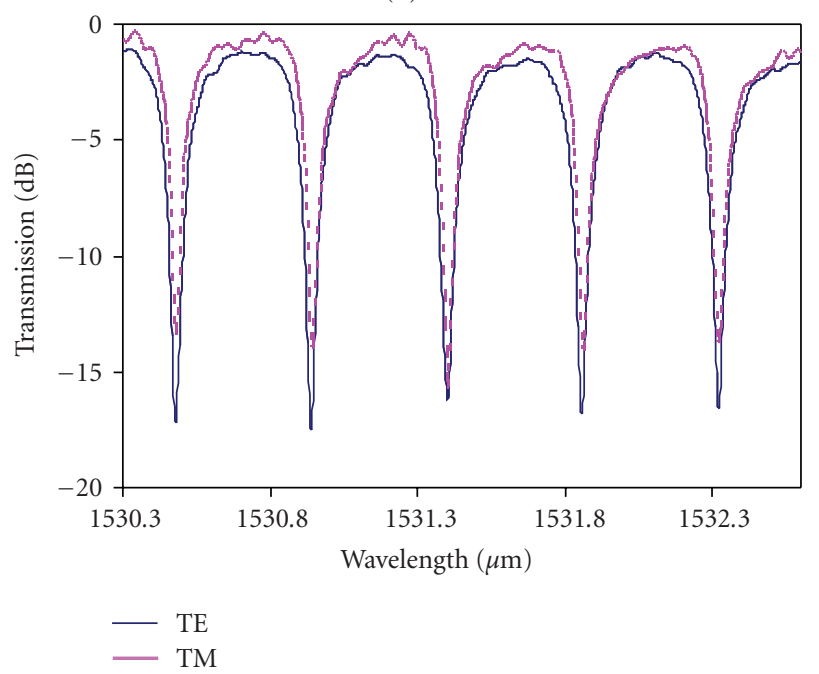

(c)

FIGURE 10: (a) SEM image of an MMI-coupled ring resonator. (b) A closeup of the MMI section. (c) TE and TM transmission spectra of the ring resonator, with an upper $\mathrm{SiO}_{2}$ cladding of $0.8 \mu \mathrm{m}$ thick and film stress of $\sigma_{\text {film }}=-250 \mathrm{MPa}$.

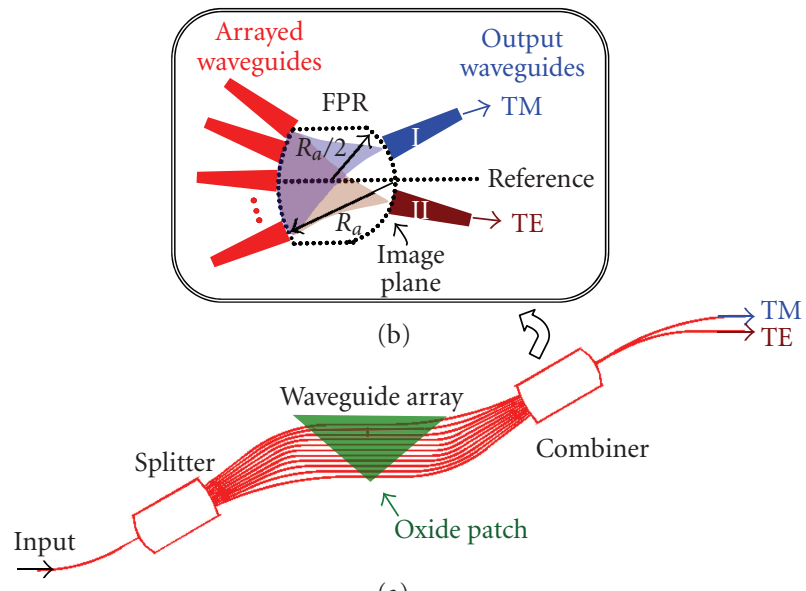

(a)

FIGURE 11: (a) Schematic layout of a broadband zero-order AWG polarization splitter. (b) Geometry of the output FPR with a Rowland circle configuration.

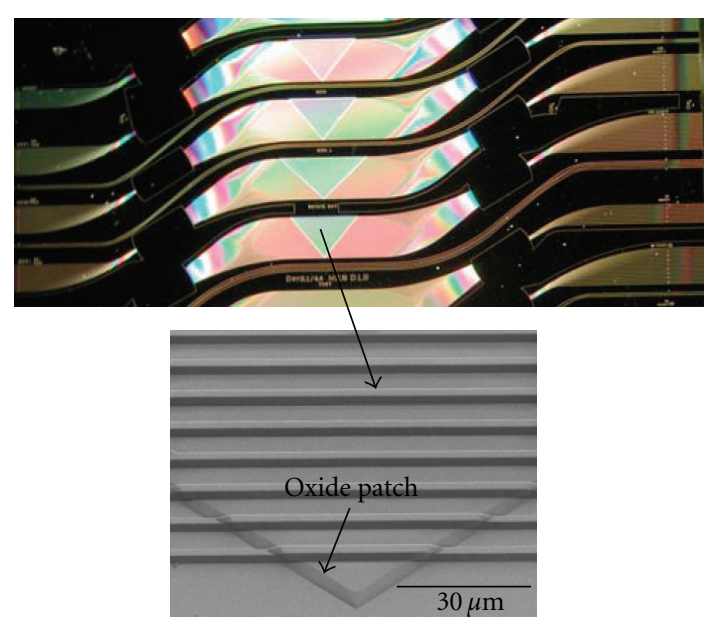

(a)

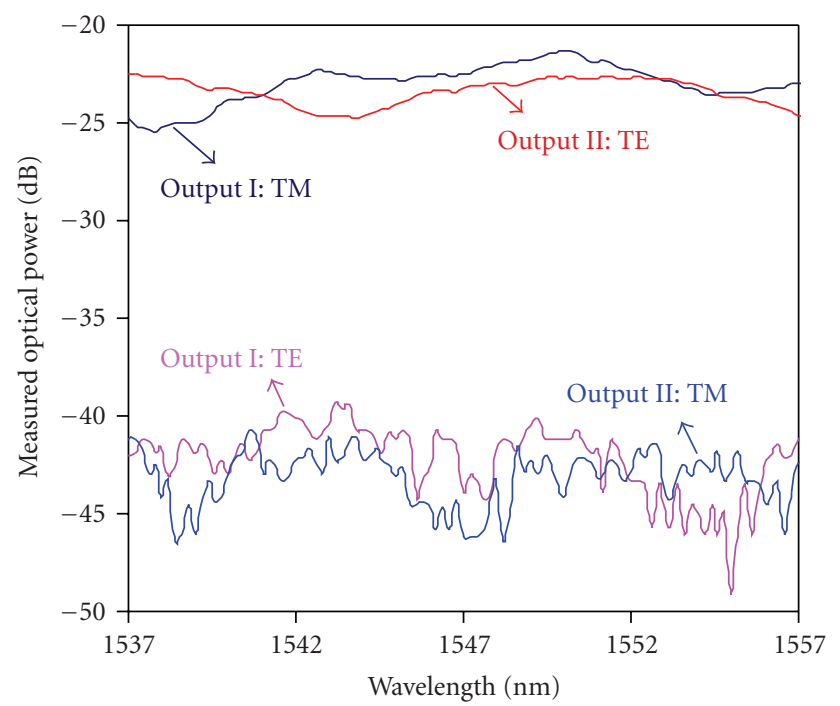

(b)

FIGURE 12: (a) A top view of the fabricated wavelength-independent zero-order AWG polarization splitters and insert an expanded SEM image of the oxide cladding patch in the waveguide array. (b) Measured transmission as a function of wavelength of a zero-order AWG-based polarization splitter.

confirmed. The measured extinction ratio of the polarization splitter was better than $-10 \mathrm{~dB}$ for both output polarizations over the entire tuning range of our laser $(1465-1580 \mathrm{~nm})$. Figure 12(b) shows the measured polarization splitter transmission for a wavelength range of $\lambda=1537$ to $1557 \mathrm{~nm}$. Over this range, the splitter extinction ratio is $<-15 \mathrm{~dB}$. The best extinction ratio achieved was $-20 \mathrm{~dB}$. Similar birefringence modification method was also applied in a Mach-Zehnder interferometer configuration to form polarization splitters and filters [33].

\section{SUMMARY}

We have reviewed the stress-induced modifications of the waveguide effective index for the TE and TM modes and the associated birefringence which are important for a wide 
range of commonly employed SOI waveguide geometries. The significance of these stress-induced effects is now being recognized in the research community and applied in component development. If not taken into consideration, these effects can lead to large deviations in device characteristics from the designed specifications. Fortunately, the use of cladding stress to correct the waveguide birefringence allows a considerable degree of freedom in designing SOI waveguides to meet other performance criteria such as relaxed dimensional tolerance, reduced loss at waveguide bends, and overall improved device performance. This technique is simple to implement, and there are no additional process steps required other than the conventional cladding layer deposition. The stress-induced modifications to the effective index in SOI waveguides are readily controlled by the stress level and the thickness of the upper-oxide cladding. We have demonstrated that cladding stress engineering can be used to achieve polarization insensitivity in AWGs, spectrometers, and ring resonators, leaving the freedom of optimizing the waveguide geometry for considerations other than the birefringence. Since the effect of the $\mathrm{SiO}_{2}$ cladding on the mode profile is negligible, there is little mode mismatch loss or polarization-dependent loss at the junctions between waveguide sections with and without the cladding. Therefore, tailored cladding patches can be applied at discrete locations in a planar waveguide circuit with negligible insertion loss and PDL penalty, as we demonstrated in the example of a broadband polarization splitter. The applications of cladding stress-induced effects can be envisioned in a variety of situations to enhance device functionality, simplify fabrication, and improve operation tolerance.

\section{ACKNOWLEDGMENT}

The authors gratefully acknowledge the contribution of Canadian Photonics Fabrication Center (CPFC) (http://cpfcccfdp.nrc-cnrc.gc.ca/) in fabricating the MMI-based polarization insensitive ring resonators.

\section{REFERENCES}

[1] L. Pavesi and D. Lockwood, Eds., Silicon Photonics, Springer, Berlin, Germany, 2004.

[2] Q. Xu, S. Manipatruni, B. Schmidt, J. Shakya, and M. Lipson, "12.5 Gbit/s carrier-injection-based silicon micro-ring silicon modulators," Optics Express, vol. 15, no. 2, pp. 430-436, 2007.

[3] A. Liu, R. Jones, L. Liao, et al., "A high-speed silicon optical modulator based on a metal-oxide-semiconductor capacitor," Nature, vol. 427, no. 6975, pp. 615-618, 2004.

[4] O. Boyraz and B. Jalali, "Demonstration of a silicon Raman laser," Optics Express, vol. 12, no. 21, pp. 5269-5273, 2004.

[5] A. Densmore, D.-X. Xu, P. Waldron, et al., "A silicon-oninsulator photonic wire based evanescent field sensor," IEEE Photonics Technology Letters, vol. 18, no. 23, pp. 2520-2522, 2006.

[6] P. Cheben, A. Bogdanov, A. Delâge, et al., "A 100-channel nearinfrared SOI waveguide microspectrometer: design and fabrication challenges," in Optoelectronic Devices and Integration, vol. 5644 of Proceedings of SPIE, pp. 103-110, Beijing, China, November 2004.
[7] R. R. Whiteman, A. P. Knights, D. George, et al., "Recent progress in the design, simulation and fabrication of small cross-section silicon-on-insulator VOAs," in Photonics Packaging and Integration III, vol. 4997 of Proceedings of SPIE, pp. 146-156, San Jose, Calif, USA, January 2003.

[8] P. Cheben, "Wavelength dispersive planar waveguide devices: echelle gratings and arrayed waveguide gratings," in Optical Waveguides: From Theory to Applied Technologies, M. L. Calvo and V. Laksminarayanan, Eds., chapter 5, p. 173, CRC Press, London, UK, 2007.

[9] S. Janz, P. Cheben, D. Dalacu, et al., "Microphotonic elements for integration on the silicon-on-insulator waveguide platform," IEEE Journal on Selected Topics in Quantum Electronics, vol. 12, no. 6, pp. 1402-1414, 2006.

[10] L. Vivien, S. Laval, B. Dumont, S. Lardenois, A. Koster, and E. Cassan, "Polarization-independent single-mode rib waveguides on silicon-on-insulator for telecommunication wavelengths," Optics Communications, vol. 210, no. 1-2, pp. 43-49, 2002.

[11] D. Dai and S. He, "Analysis of the birefringence of a siliconon-insulator rib waveguide," Applied Optics, vol. 43, no. 5, pp. 1156-1161, 2004.

[12] W. R. Headley, G. T. Reed, F. Gardes, A. Liu, and M. Paniccia, "Enhanced polarisation-independent optical ring resonators on silicon-on-insulator," in Optoelectronic Integration on Silicon II, vol. 5730 of Proceedings of SPIE, pp. 195-202, San Jose, Calif, USA, January 2005.

[13] D.-X. Xu, P. Cheben, D. Dalacu, et al., "Eliminating the birefringence in silicon on insulator ridge waveguides using the cladding stress," Optics Letters, vol. 29, no. 20, pp. 23842386, 2004.

[14] M. Huang, "Stress effects on the performance of optical waveguides," International Journal of Solids and Structures, vol. 40, no. 7, pp. 1615-1632, 2003.

[15] I. Kiyat, A. Aydinli, and N. Dagli, "Polarization characteristics of compact SOI rib waveguide racetrack resonators," IEEE Photonics Technology Letters, vol. 17, no. 10, pp. 2098-2100, 2005.

[16] W. N. Ye, D.-X. Xu, S. Janz, et al., "Birefringence control using stress engineering in silicon-on-insulator (SOI) waveguides," Journal of Lightwave Technology, vol. 23, no. 3, pp. 1308-1318, 2005.

[17] D.-X. Xu, W. N. Ye, A. Bogdanov, et al., "Design of polarization-insensitive components using geometrical and stress-induced birefringence in SOI waveguides," in Optoelectronic Integration on Silicon II, vol. 5730 of Proceedings of SPIE, pp. 158-172, San Jose, Calif, USA, January 2005.

[18] D.-X. Xu, S. Janz, and P. Cheben, "Design of polarizationinsensitive ring resonators in silicon-on-insulator using MMI couplers and cladding stress engineering," Photonics Technology Letters, vol. 18, no. 2, pp. 343-345, 2006.

[19] D.-X. Xu, P. Cheben, A. Delâge, et al., "Polarization-insensitive MMI-coupled ring resonators in silicon-on-insulator using cladding stress engineering," in Silicon Photonics II, vol. 6477 of Proceedings of SPIE, pp. 1-11, San Jose, Calif, USA, January 2007.

[20] W. N. Ye, D.-X. Xu, S. Janz, P. Waldron, P. Cheben, and N. G. Tarr, "Passive broadband silicon-on-insulator polarization splitter," Optics Letters, vol. 32, no. 11, pp. 1492-1494, 2007.

[21] A. Kilian, J. Kirchhof, B. Kuhlow, G. Przyrembel, and W. Wischmann, "Birefringence free planar optical waveguide made by flame hydrolysis deposition (FHD) through tailoring of the overcladding," Journal of Lightwave Technology, vol. 18, no. 2, pp. 193-198, 2000. 
[22] C. K. Nadler, E. K. Wildermuth, M. Lanker, W. Hunziker, and H. Melchior, "Polarization insensitive, low-loss, low-crosstalk wavelength multiplexer modules," IEEE Journal on Selected Topics in Quantum Electronics, vol. 5, no. 5, pp. 1407-1412, 1999.

[23] T. S. Narasimhamurty, Photoelastic and Electro-Optic Properties of Crystals, Plenum Press, New York, NY, USA, 1981.

[24] B. E. Little, S. T. Chu, H. A. Haus, J. Foresi, and J.-P. Laine, "Microring resonator channel dropping filters," Journal of Lightwave Technology, vol. 15, no. 6, pp. 998-1005, 1997.

[25] C. K. Madsen, "Efficient architectures for exactly realizing optical filters with optimum bandpass designs," IEEE Photonics Technology Letters, vol. 10, no. 8, pp. 1136-1138, 1998.

[26] Q. Xu, B. Schmidt, S. Pradhan, and M. Lipson, "Micrometerscale silicon electro-optic modulator," Nature, vol. 435, pp. 325-327, 2005.

[27] D.-X. Xu, A. Densmore, P. Waldron, et al., "High bandwidth SOI photonic wire ring resonators using MMI couplers," Optics Express, vol. 15, no. 6, pp. 3149-3155, 2007.

[28] A. Yariv, "Universal relations for coupling of optical power between microresonators and dielectric waveguides," Electronics Letters, vol. 36, no. 4, pp. 321-322, 2000.

[29] L. B. Soldano and E. C. M. Pennings, "Optical multi-mode interference devices based on self-imaging: principles and applications," Journal of Lightwave Technology, vol. 13, no. 4, pp. 615-627, 1995.

[30] P. A. Besse, E. Gini, M. Bachmann, and H. Melchior, "New $2 \times 2$ and $1 \times 3$ multimode interference couplers with free selection of power splitting ratios," Journal of Lightwave Technology, vol. 14, no. 10, pp. 2286-2292, 1996.

[31] T. Saida, A. Himeno, M. Okuno, A. Sugita, and K. Okamoto, "Silica-based $2 \times 2$ multimode interference coupler with arbitrary power splitting ratio," Electronics Letters, vol. 35, no. 23, pp. 2031-2033, 1999.

[32] M. Bachmann, P. A. Besse, and H. Melchior, "General selfimaging properties in $\mathrm{N} \times \mathrm{N}$ multimode interference couplers including phase relations," Applied Optics, vol. 33, no. 18, pp. 3905-3911, 1994.

[33] W. N. Ye, D.-X. Xu, S. Janz, P. Waldron, and N. G. Tarr, "Stress-induced SOI polarization splitter based on MachZehnder Interferometers (MZI)," in Proceedings of the 3rd IEEE International Conference on Group IV Photonics (GFP '06), pp. 249-251, Ottawa, Canada, September 2006. 

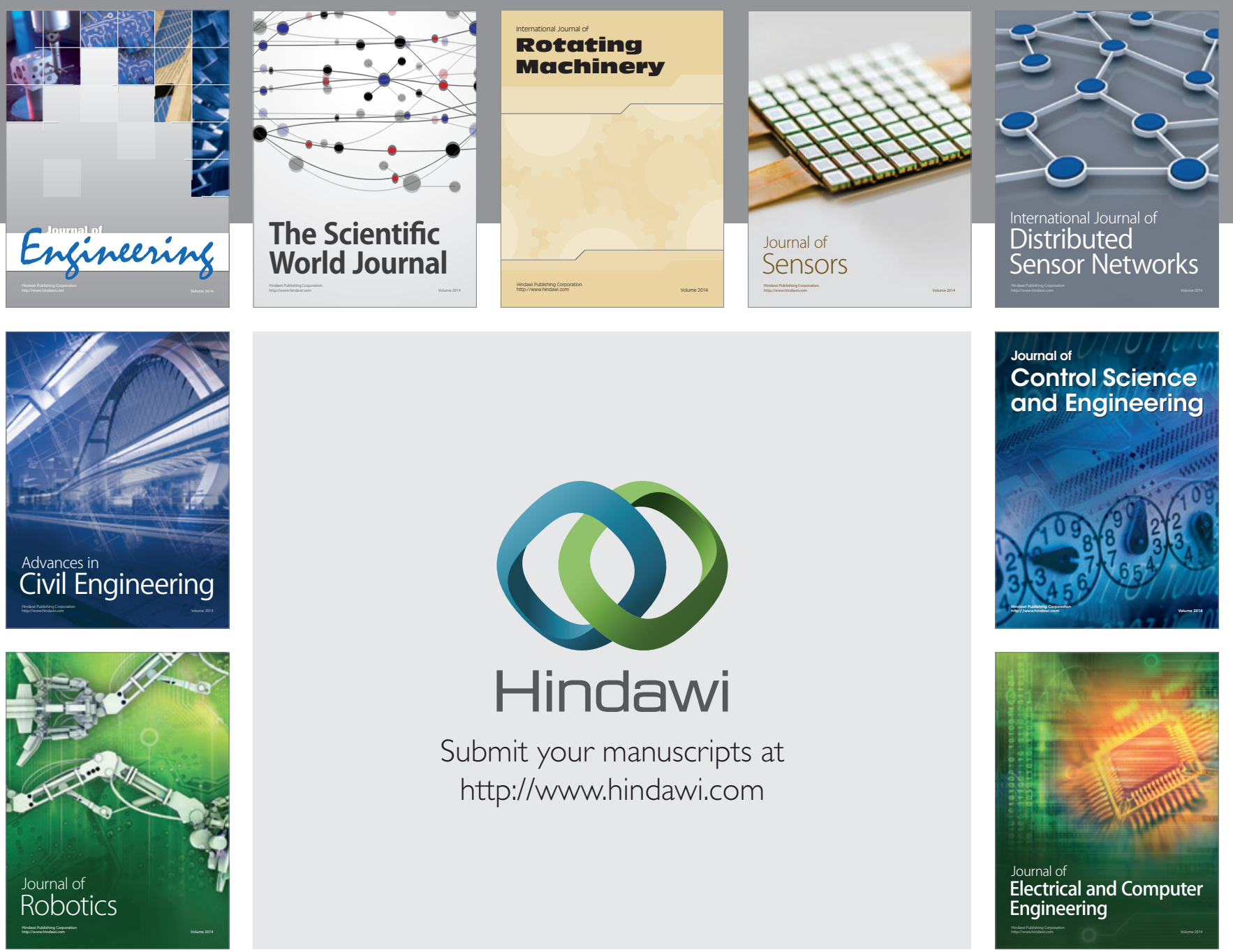

Submit your manuscripts at

http://www.hindawi.com
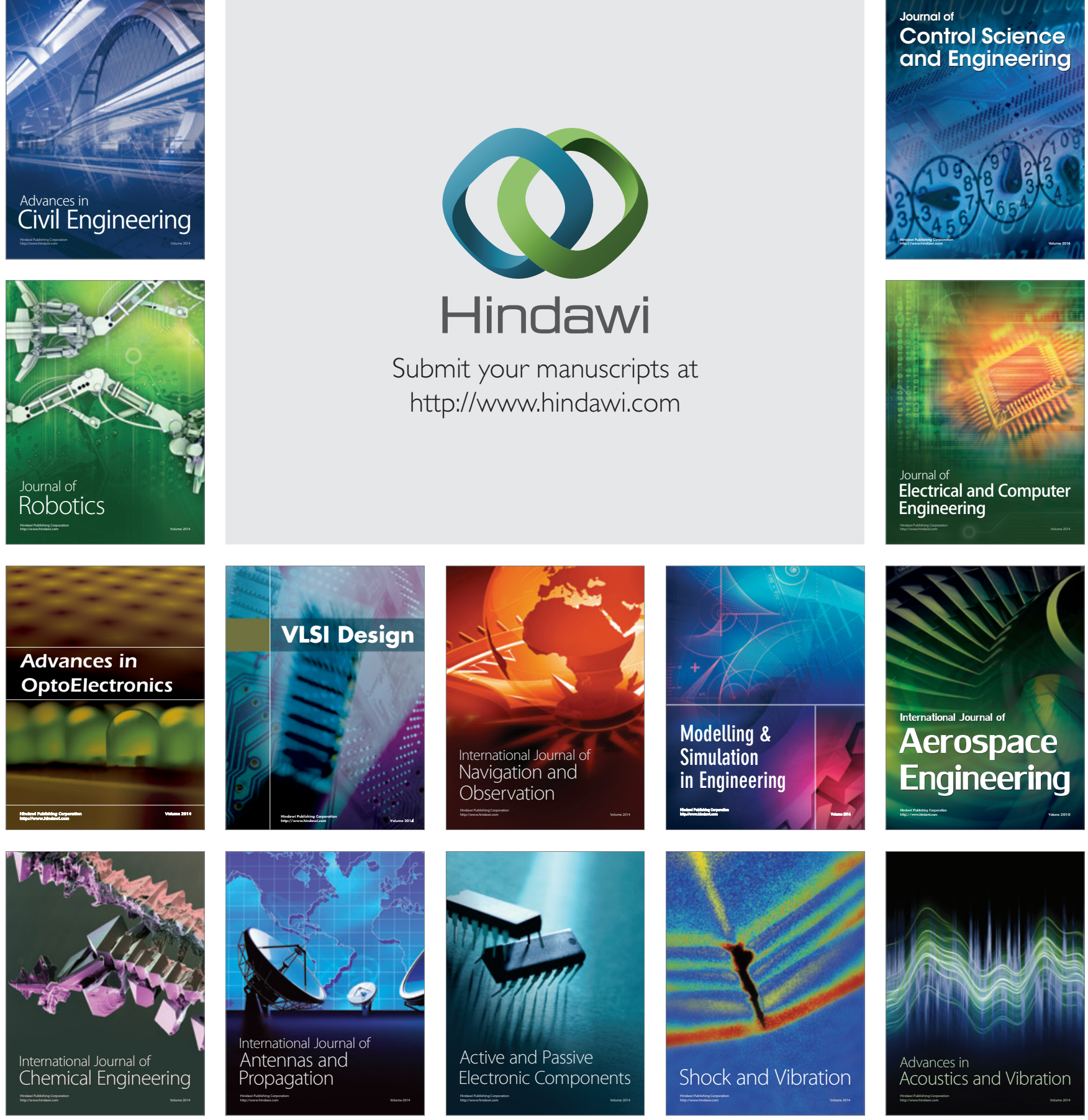